\title{
Coronal and Solar Wind Elemental Abundances
}

\author{
J. C. Raymond*, J. E. Mazur ${ }^{\dagger}$, F. Allegrini**, E. Antonucci ${ }^{\ddagger}$, G. Del Zanna ${ }^{\S}$, \\ S. Giordano ${ }^{\ddagger}$, G. Ho ${ }^{\Uparrow}$, Y.-K. Ko* ${ }^{*}$ E. Landi ${ }^{\|}$, A. Lazarus ${ }^{\dagger \dagger}$, S. Parenti ${ }^{\ddagger}$, G. Poletto ${ }^{\S \S}$, \\ A. Reinard ${ }^{\text {ITा }}$ J. Rodriguez-Pacheco ${ }^{* * *}$, L. Teriaca ${ }^{\S \S}$, P. Wurz ${ }^{* *}$ and L. Zangrillit ${ }^{\dagger \dagger}$ \\ ${ }^{*}$ Center for Astrophysics, 60 Garden St., Cambridge, MA 02138, USA \\ ${ }^{\dagger}$ The Aerospace Corporation, El Segundo, CA 90245, USA \\ ${ }^{* *}$ Physikalisches Institut, University of Bern, sidlerstrasse 5, CH-3012 Bern, Switzerland \\ ${ }^{\ddagger}$ Osservatorio Astronomico di Torino, 10025 Pino, Torinese, Italy \\ ${ }^{\S}$ DAMTP, University of Cambridge UK, Cambridge, UK \\ "Johns Hopkins University, Applied Physics Laboratory, Johns Hopkins Road, Laurel, MD 20723 USA \\ $\|_{\text {Naval Research Laboratory, Washington, DC USA }}$ \\ ${ }^{\dagger}$ MIT, Room 37-687, 77 Massachusetts Avenue, Cambridge, MA 02139 USA \\ $\$$ Centre for Astrophysics, University of Central Lancashire, Preston, UK \\ ${ }_{\$}$ Arcetri Astrophysics Observatory, Largo Enrico Fermi 5, Firenze 50125, Italy \\ ${ }^{11}$ Department of Atmospheric, Oceanic, and Space Science, University of Michigan, Ann Arbor, MI 48109 USA \\ ${ }^{* * *}$ Departamento Fisica - Universidad Alcala, Alcala de Henares, Madrid 28871 Spain \\ ${ }^{\dagger}$ Dipartimento di Astronomia e Scienza dello Spazio, Universita di Firenze, Largo Enrico Fermi 5, Firenze \\ 50125 Italy
}

\begin{abstract}
Coronal elemental abundances, as compared with abundances in the solar wind and solar energetic particles, provide the means for connecting solar wind gas with its coronal source. Comparison of coronal abundances with photospheric values shows fractionation with the ionization potential of the atom, providing important, though not yet fully understood, information about the exchange of material between corona and chromosphere. Fractionation due to gravitational settling provides clues about flows within the corona.

In this paper, we discuss the uncertainties of abundance determinations with spectroscopic techniques and in situ measurements, we survey the ranges of abundance variations in both the corona and solar wind, and we discuss the progress in correlating solar wind features with their coronal sources.
\end{abstract}

\section{INTRODUCTION}

Elemental abundances in the solar corona are the basis of comparison for investigations of the coronae of other stars and for abundances measured in the solar wind. They differ from solar photospheric abundances by as much as an order of magnitude, and they vary from place to place and time to time. Fractionation according to the ionization potential of the neutral atom (First Ionization Potential, or FIP) is ubiquitous, and evidence for gravitational settling in quiescent streamers has been reported. Measurements of abundances in the solar wind show similar variations, strongly correlated with the speed of the wind. Abundances of solar energetic particles show variations in isotopic ratios indicating strong mass fractionation.

Abundance variations are useful as means of connecting features in the corona with solar wind structures that arise from them. They have enormous potential as tools for understanding the physical processes in the chromosphere that give rise to the wind through analysis of the FIP effect. Preferential heating of various ion species [1] and the effects of ion drag in the accelerating wind [2] will eventually be useful for determining the physical conditions and processes in the region out to a few solar radii where the wind forms.

To realize this potential, we must understand and exploit the variations of coronal and solar wind abundances. We must understand and reduce the uncertainties in both the analysis of spectroscopic observations of the corona and the measurement of abundances in the solar wind. We must be able to reliably connect structures in the solar wind with their coronal sources. The following sections discuss the uncertainties, the variations and the connection between solar and solar wind features, respectively.

CP598, Solar and Galactic Composition, edited by R. F. Wimmer-Schweingruber (C) 2001 American Institute of Physics 0-7354-0042-3/01/\$18.00 


\section{UNCERTAINTIES IN SPECTROSCOPIC ESTIMATES OF CORONAL ABUNDANCES}

Before SOHO, remote sensing instruments revealed that different solar features had very different elemental compositions. Most of these results were obtained using the Skylab S0-82A observations (see e.g., [3]) that were limited by the fact that only a few lines could be used, given the characteristics of the overlapping spectroheliograms. Now, the spectroscopic instruments on board SOHO provide new opportunities to study in much greater detail the chemical composition of the solar transition region and corona. These instruments provide better radiometric calibration, much higher spatial and spectral resolution, and coronagraphic capabilities. However, it is important to be aware of the other factors that limit the accuracy of coronal abundance determinations.

Del Zanna et al. [4] reviewed the main factors that affect the determination of the element abundances from spectroscopic instruments that observe the low corona, such as CDS and SUMER on board SOHO. The element abundances that are derived depend on:

a) The spectroscopic method used. The method of estimating the emission measure distribution as a function of temperature can affect the results, particularly if only a few spectral lines are available.

b) The atomic data and the ionization equilibrium calculations that are adopted. We discuss these below.

c) Temperature and density effects. Many analyses use, for instance, an ionization model computed for the low density limit, while at transition region densities some dielectronic recombination ratios are reduced by about a factor of 2. In the case of spectroscopic instruments that observe the outer corona (such as UVCS) temperature and density effects are reduced because the coronal plasma is nearly isothermal and the density is low. On the other hand, other effects such as the photoexcitation become important, and have to be taken into account.

d) Instrument calibration. For many purposes only the relative sensitivity as a function of wavelength needs to be known. For modern UV and EUV instruments, this should be calibrated to $15 \%$ or better before launch.

Several approximations are commonly made, often tacitly. Proton collisional excitation and de-excitation processes are usually neglected. They are generally most important for fine structure transitions. Stimulated emission and absorption of ambient radiation are often neglected, though they can be important for hydrogen thanks to its metastable $2 \mathrm{~s}$ level [5]. The optical depth to resonance scattering is generally ignored because of the geometrical complexity introduced by scattering, but it may be important in some cases $[6,7]$.
Various authors (see, e.g., $[8,9,10])$ have pointed out that all of these factors may have led to inaccurate determinations of the element abundances in the past. Del Zanna et al. [4] show examples where in particular cases these factors do indeed lead to variations of a factor of two or more in the derived element abundances. Since each of these variations is of the same order as the FIP effect that we want to measure, these factors should be given full consideration.

\subsection{Atomic Rates}

Any plasma diagnostic technique involving EUV line intensities requires the knowledge of a large amount of atomic data and transition probabilities in order to be carried out; these are necessary to calculate the theoretical line intensities for a given ion to be compared with the observations. Any uncertainty or inaccuracy in these atomic data can have significant impact on the diagnostic results.

The number of photons emitted in an optically thin spectral line $i \rightarrow j$ is given by

$$
I_{i j}=\frac{1}{4 \pi} \int_{h} N_{j}\left(X^{+m}\right) A_{j i} d h \quad p h \mathrm{~cm}^{-2} s^{-1} s r^{-1}
$$

The Contribution Function $\mathrm{G}\left(T, N_{e}\right)$ of the line is defined as

$$
G\left(T, \lambda_{i, j}\right)=\frac{N_{j}\left(X^{+m}\right)}{N\left(X^{+m}\right)} \frac{N\left(X^{+m}\right)}{N(X)} \frac{N(X)}{N(H)} \frac{N(H)}{N_{e}} \frac{A_{j i}}{N_{e}}
$$

where $\frac{N_{j}\left(X^{+m}\right)}{N\left(X^{+m}\right)}$ is the relative upper level population; $\frac{N\left(X^{+m}\right)}{N(X)}$ is the relative abundance of the ion $X^{+m}$ (ion fraction); $\frac{N(X)}{N(H)}$ is the abundance of the element $X$ relative to hydrogen; $\frac{N(H)}{N_{e}}$ is the hydrogen abundance relative to the electron density; and, $A_{j i}$ is the Einstein coefficient for spontaneous emission.

The Differential Emission Measure (DEM) is defined so that

$$
\varphi(T)=N_{e}^{2} \frac{d h}{d T}
$$

the number of photons emitted in a spectral line may be expressed as

$$
I_{i j}=\frac{1}{4 \pi} \int_{T} G\left(T, \lambda_{i, j}\right) \varphi(T) d T
$$

$>$ From Equation 2 it is possible to identify the main sources of uncertainties in the evaluation of the $\mathrm{G}\left(T, N_{e}\right)$ functions. 


\subsection{Relative upper level population}

The relative population $\frac{N_{j}\left(X^{+m}\right)}{N\left(X^{+m}\right)}$ must be calculated by solving the statistical equilibrium equations for a number of low lying levels and including all the important collisional and radiative excitation and de-excitation mechanisms.

Stimulated and spontaneous radiative transition probabilities are mostly obtained from $a b$ initio theoretical calculations, using programs such as SUPERSTRUCTURE [11], or CIV3 [12].

The electron collisional excitation rate coefficient $\left(\mathrm{cm}^{3} \mathrm{~s}^{-1}\right)$ for a Maxwellian electron velocity distribution with a temperature $T_{e}(\mathrm{~K})$, is given by:

$$
C_{i, j}^{e}=\frac{8.63 \times 10^{-6}}{T_{e}^{1 / 2}} \frac{\Upsilon_{i, j}\left(T_{e}\right)}{\omega_{i}} e^{\left(-E_{i, j} / k T_{e}\right)}
$$

where $\omega_{i}$ is the statistical weight of level $i ; E_{i, j}$ is the energy difference between levels $i$ and $j ; k$ is the Boltzmann constant and $\Upsilon_{i, j}$ is the thermally-averaged collision strength:

$$
\Upsilon_{i, j}\left(T_{e}\right)=\int_{0}^{\infty} \Omega_{i, j} e^{\left(-E_{j} / k T_{e}\right)} d\left(E_{j} / k T_{e}\right)
$$

where $\Omega$ is the collision strength and $E_{j}$ is the energy of the scattered electron relative to the final energy state of the ion. Non-Maxwellian electron distributions can also be considered. However, the effect of a non-Maxwellian tail on collisional excitation rates is generally small, because the excitation rate is usually dominated by electron energies below 2 or $3 \mathrm{kT}$.

Collision strengths are obtained from theoretical calculations. The solution of the electron-ion scattering problem is complex and requires extensive computing resources. The accuracy of a particular calculation depends on two main factors. The first is the representation which is used for the target wavefunctions, the second is the type of scattering approximation chosen. The target must take account of configuration interaction and allow for intermediate coupling for the higher stages of ionization. The main approximations used for electron-ion scattering are Distorted Wave (DW) [13], Coulomb Bethe (CBe) [14] and the more elaborate Close-Coupling (CC) [15]. Laboratory measurements of the cross sections are available for some transitions, and they provide an important benchmark for the theoretical calculations.

Comprehensive databases that include the best atomic data are becoming available. Besides making new data quickly available to researchers, they promote consistency among various analyses. In solar physics, CHIANTI [16] is most popular. Recently, CHIANTI has been extended to cover the X-ray wavelengths [18]. Other databases such as APEC/APED [17] emphasize X-ray wavelengths.

The accuracy of theoretical calculations for the radiative and collisional transition probabilities is of crucial importance for the determination of relative level populations. However, there is no direct way of assessing the accuracy of these theoretical calculations, and in the literature the authors limit themselves to quoting an accuracy of usually $10 \%$ for radiative transition probabilities, and of $30 \%$ for collisional transition probabilities.

However, the accuracy quoted for the collisional transition probabilities is sometimes very optimistic. In fact, the increasing computing power of modern computers allows the inclusion of more and more complex atomic models and to take into account interactions between a larger number of terms. The presence of these previously neglected configurations, terms and interactions can lead to very different results in the collision rates. Examples are given for Fe XII [19], for Fe XIV [20] and for Fe IX [21]. In these cases, the effects of more accurate calculations go beyond the $30 \%$ accuracy quoted by previous authors, and have a strong impact in the diagnostic results obtained from line intensity ratios. However, Fe IX, XII and XIV are probably the worst cases. Cascades from higher-lying levels are also important in many cases, such as Fe XVII [22].

One way to evaluate the consistency of the atomic data is to model a set of observational data containing many spectral lines and examine the scatter in ratios of observed to predicted fluxes. The scatter will include measurement and calibration errors, along with any inappropriate assumptions of the model, but it gives a general indication of the accuracy of the atomic rates. Spectra from SERTS observations of a solar active region [23] between 171 and $445 \AA$ have been especially useful in this regard $[24,25,26]$, and they demonstrate the improvements in recent years incorporated into the CHIANTI database. More recently, [27] and [10] have carried out a similar comparison using an off-limb observation of a streamer obtained with SUMER [29] between 800 and $1600 \AA$. This comparison involves mainly intercombination and forbidden lines within the ground configuration for a number of coronal ions. Landi \& Feldman [27] find in general good agreement between the CHIANTI database and observed line intensities.

\subsection{Ion fractions}

It is usually assumed that the plasma is in ionization equilibrium; this assumption, which can be misleading in highly dynamic plasma, allows the use of the ion fraction datasets found in the literature. It is not difficult to compute time-dependent ionization states for any specified 
model of temperature and density (e.g., [30]), but there is rarely a unique model available for a given data set. The equilibrium ion fractions available in the literature have been calculated using the state-of-the-art ionization and recombination rates available at the time of publication. However, progress in the theoretical models for ionization and recombination processes has led to significant changes in ionization and recombination rates. The tables of Mazzotta et al. [31] reflect the best information available to date.

An assessment of the quality of ion abundance computations is not an easy task, due to the complicated and largely unknown temperature structure of the solar atmosphere as seen along the line of sight. In the recent past, Masai [32] investigated the impact of uncertainties in the ionization and recombination rates on X-ray spectral analyses, finding that differences in the rates led to significant differences in iron abundance and plasma temperature measurements. Phillips \& Feldman [33] have used Yohkoh flare observations to check the ion fractions of He-like ions, concluding that the observed spectra were consistent with the adopted ion fractions at the $50 \%$ level of precision, and this led to changes to the plasma diagnostic results. Phillips \& Feldman suggest that Arnaud \& Rothenflug [34] ion abundances for [S XV] and [Ca XIX] need to be improved.

Young \& Mason [28] used SOHO/CDS spectral data to study element abundance variations. They found enhancements of up to a factor of 5 for Ca X, but suggested that this was due to inaccurate ionization fraction calculations. Del Zanna [26] has also used SOHO/CDS observations to point out disagreements between lines of the $\mathrm{Li}$ and $\mathrm{Na}$ isoelectronic sequences, compared to the lines of all the other sequences. Del Zanna [26] has also shown that some of these discrepancies, including the $\mathrm{Ca} \mathrm{X}$ one, are indeed related to inaccurate ionization equilibrium calculations, since they are resolved with the use of more recent calculations [31].

More recently, [35] investigated the effect of changing ion fractions datasets on temperature diagnostics. They used the spectra from an isothermal region outside the solar limb, and adopted in turn the ionization equilibria from Shull \& Van Steenberg [36], Arnaud \& Rothenflug [34] incorporating the latest revisions to the iron ions by Arnaud \& Raymond [37] and Mazzotta et al. [31]. They found negligible differences in the results, showing that in the case of an isothermal spectrum the choice of the ion fractions has little effect on the temperature diagnostic results. Unfortunately, they did not investigate the effect on abundance diagnostics. Gianetti et al. [38] carried out a similar study using active region observations on the disk. They found that the choice of the ion fractions has a large effect on the diagnostic results, both in terms of DEM and element abundance measurements.

It is important to recognize that improved abundance determinations in recent years result in almost equal measure from the efforts of laboratory and theoretical atomic physicists, the calibration efforts of the SOHO instrument teams, and the careful planning and data analysis of SOHO observers.

\section{UNCERTAINTIES OF SOLAR WIND AND SOLAR ENERGETIC PARTICLE ABUNDANCE MEASUREMENTS}

Abundances can be measured both in the normal solar wind and in Solar Energetic Particle (SEP) events. As for spectroscopic measurements, instrument calibration is a crucial part of the analysis. Modern instruments such as SWICS/ULYSSES [39], CELIAS/SOHO [40] and SWIMS and SWICS/ACE [41] can measure solar wind abundances with high sensitivity and resolution of mass and charge. Instruments such as ACE/ULEIS and ACE/SIS measure higher energy particles (100 keV/nuc to $10 \mathrm{MeV} /$ nuc), again with better sensitivity and resolution than earlier generations of instruments. With instruments such as these it is possible to measure the abundances of rare species and to measure isotopic abundance ratios. The sensitivity to particles of different mass is calibrated before launch, and sometimes extensive calibration campaigns are performed even after launch using the flight-spare instruments. In recent publications on solar wind abundances the quoted errors are around $10 \%$ (or larger for elements of very low abundance) and as low as $5 \%$ for isotopic ratios.

One important limitation, as for spectroscopic instruments, is dynamic range. This is especially an issue when comparing hydrogen and helium to other species, as protons and alpha particles are usually measured with a different sensor than that used for the heavy elements. Thus most papers quote ratios of different elements to oxygen or silicon, but the ratios relative to hydrogen are not often determined directly. Ratios relative to hydrogen are given by Wimmer-Schweingruber [42] for the fast and slow solar wind. They imply that the FIP effect is an enhancement of low-FIP elements rather than a depletion of high-FIP elements relative to hydrogen. For gradual SEP events corresponding to particles accelerated by Coronal Mass Ejection (CME) shocks in the corona, Reames [43] gives a H/O ratio of $1570 \pm 220$ (statistical uncertainty), within about $16 \%$ of the photospheric value. For impulsive SEP events (corresponding to particles accelerated in flares) the ratio is somewhat lower, but less well determined.

SEPs can be measured with very good mass resolution, as low as $\sigma_{m}>0.3 \mathrm{amu}$ [44]. Isotopic abundance measurements can be reliably performed. The main limitation is that acceleration processes and transport effects 
lead to fractionation in mass or mass/charge (Reames [43]; see section 6).

\section{ABUNDANCE VARIATIONS IN THE CORONA}

Most astronomers and solar wind physicists tend to assume that there is one set of photospheric abundances and one set of coronal abundances, differing by a FIP fractionation of a factor of 3-4. The tables of [45] are the current standard. A single set of photospheric abundances is probably a good approximation, though the 'standard' abundance set continues to evolve. This is especially troublesome for elements such as neon which lack strong lines at optical wavelengths. The most important change in recent years is the decrease in $\mathrm{O}$ abundance by 0.2 dex [45], as many relative abundance measurements are scaled to oxygen. (See also Holweger, these proceedings, and references therein.)

Substantial FIP abundance variations within the corona were established some time ago, but the fragmentary nature of the results (often just the ratio of one element to one other), disputes about whether erroneous atomic data might account for some of the apparent variations, and the large range of abundances without a clear pattern have clouded the issue. Abundance variations in solar flares (e.g., [46]) are believed to result from evaporation of chromospheric material into loops filled with pre-existing coronal plasma. The large number of spectral lines available to the spectrographs aboard SOHO have begun to clarify the situation. Some results for the FIP bias are listed in Table 1. While the expected factor of about 3-4 is often observed in the quiet Sun, the FIP bias is only half as large in coronal holes, and it can be up to twice as large in active regions. Coronal hole plumes do not show extreme FIP effects [47]. Earlier reports of extreme FIP enhancement can be explained in terms of their emission in a narrow temperature band [4].

Off-limb observations in the UV open the possibility of measuring abundances relative to hydrogen. UVCS observations of a strong depletion of $\mathrm{O}$ in the core of the equatorial streamer at solar minimum were interpreted in terms of gravitational settling [48], and SUMER observations of a strong decline of iron lines with height compared with the decline of silicon lines support this idea. Parenti et al. [49] derived the oxygen abundances in a streamer core and edges from UVCS data taken in June 2000. The absolute abundance of oxygen turned out to be $\sim 8.6$ on the edges and 8.4 in the core. The electron temperature derived from line ratio techniques appears to decrease by $15 \%$ from the core to the edges. Zangrilli, Poletto and Biesecker [50] studied the oxygen abundance in streamers in July 1996 between 2.5 and 4 $R_{\odot}$. Two streamers at the minimum of solar activity were analyzed (6 and 11 July 1996) and the oxygen abundance was found to vary from about 8.0 in the core to 8.4 in the legs of the streamer (about $10^{\circ}$ above the equator). Zangrilli et al. also found a hint of abundance decrease with height (about $50 \%$ between 2.5 and $5 R_{\odot}$ ) in the core. At these heights there may be some departure from ionization equilibrium. Marrocchi et al [51] found a $40 \%$ decline in oxygen abundance in a streamer between 1.5 and $2.2 R_{\odot}$.

It is more difficult to obtain abundances in coronal holes, because the lines are fainter and because the assumption of ionization equilibrium is problematic. An attempt to determine the oxygen abundance in interplume lanes has been performed by Teriaca et al [52]. From coronal hole O VI doublet data taken by SUMER in 1996 , the authors infer an oxygen abundance $\geq 8.50$ depending on the adopted density profile. Antonucci et al. [53] used UVCS observations of solar minimum coronal holes to infer an oxygen abundance consistent with photospheric and ULYSSES values.

Among the most difficult, but most important, measurements is the helium abundance. Laming and Feldman [54] find $\mathrm{He} / \mathrm{H}$ ratio of 0.05 in both a streamer and coronal hole near solar minimum.

\section{ABUNDANCE VARIATIONS IN THE SOLAR WIND}

The variations in $\mathrm{He} / \mathrm{H}$ and $\mathrm{FIP}$ bias between fast and slow solar wind are well established [67, 68], and solar physicists tend to view solar wind abundances as bimodal. The FIP bias is modest (around 1.5) in the fast wind and 3-4 in the slow wind. While this does seem to be a good approximation for the average abundances, there are short time-scale variations.

Heavy ions in the solar wind (e.g., O, Si, Fe) and helium generally show a much larger variability in their densities than the protons. Typically the variability is larger by a factor of 10 . This variability has been observed for helium with respect to protons already a while ago as has been reviewed by Neugebauer [69]. She found that the ratio of protons to alpha particles in the solar wind $\left(n_{p} / n_{a}\right)$ varies in the range from $8.1 \times 10^{-4}$ to $4.17 \times 10^{-1}$, a variation by a factor of 500 . For heavy elements this variability is illustrated in Figure 1 where two commonly used abundance ratios $\mathrm{Fe} / \mathrm{O}$ and $\mathrm{Si} / \mathrm{O}$ are plotted for a period of 80 days during solar minimum in 1996. This time period also contains short coronal hole solar wind sections of about one day since the "elephant trunk" coronal hole passed three times during that period. One can easily see the large fluctuations in the abun- 
TABLE 1.

\begin{tabular}{|llccc|}
\hline Structure & Paper & Height & Instrument & FIP Bias \\
\hline Coronal Hole & Doschek et al. 98 [55] & $1.0-1.2 R_{\odot}$ & SUMER & $<2$ \\
Coronal Hole & Feldman et al. 98 [56] & $1.03-1.5 R_{\odot}$ & SUMER & $<1.3$ \\
Coronal Hole Plume & Young et al. 99 [57] & $1.0-1.1 R_{\odot}$ & CDS & 1.5 \\
\hline Quiet Sun & Warren 99 [58] & $1.05-1.35 R_{\odot}$ & SUMER & $2.3 \pm 0.7$ \\
Quiet Sun & Laming et al. 99 [59] & $1.1 R_{\odot}$ & SUMER & $3-4$ \\
Quiet Sun & Young \& Mason 98 [28] & Disk & CDS & 2 \\
\hline EQ Streamer core & Raymond et al. 97 [48] & $1.5 R_{\odot}$ & UVCS & $3-4$ \\
EQ Streamer legs & Raymond et al. 97 [48] & $1.5 R_{\odot}$ & UVCS & $3-4$ \\
EQ Streamer base & Feldman et al. 99 [56] & $1.03-1.5 R_{\odot}$ & SUMER & 4 \\
EQ Streamer & Parenti et al. 00 [61] & $1.6 R_{\odot}$ & UVCS & 3 \\
EQ Streamer base & Parenti et al. 00 [61] & $1.02-1.19 R_{\odot}$ & CDS & 1.1 \\
\hline Active Region & Young \& Mason 98 [28] & $1.5 R_{\odot}$ & CDS & $1-9$ \\
Active Region & Rank et al. 99 [62] & Disk & CDS & $5-9$ \\
Active Region & Dwivedi et al. 99 [63] & $1.04-1.11 R_{\odot}$ & SUMER & 8 \\
Active Region & Ko et al. 01 [64] & $1.3-1.7 R_{\odot}$ & UVCS & 4 \\
\hline CME & Ciaravella et al. 97 [65] & $1.5 R_{\odot}$ & UVCS & 1 \\
\hline Prominence & Spicer et al. 98 [66] & $1.5 R_{\odot}$ & SUMER & 2 \\
\hline
\end{tabular}

dance ratios exceeding the values for the FIP fractionation by an order of magnitude. Of course, some of the spikes in Figure 1 are of instrumental or statistical nature. This high variability averages out when investigating longer time periods. Analysis of the data presented in Figure 1 showed that the established heavy element abundances for $\mathrm{Fe} / \mathrm{O}$ and $\mathrm{Si} / \mathrm{O}$ are obtained including the FIP fractionation pattern when sufficiently long averages are performed [70]. These short-time variations in the abundance of heavy ions in the solar wind are thought to be caused in the corona and are believed to be of temporal and spatial nature [71]. Similar short-time fluctuations are also observed for the charge states of heavy elements [72, 73], although the amplitude of the variations is smaller.

\section{ABUNDANCE VARIATIONS IN SOLAR ENERGETIC PARTICLE EVENTS}

Transport effects can be a significant source of abundance variations in individual SEP events associated with interplanetary shocks. The basic parameter that describes a particle's motion in the interplanetary medium is its rigidity, or momentum per unit charge. Rigidity is proportional to the mass to charge $(\mathrm{A} / \mathrm{Q})$ ratio, and it is the case that $\mathrm{A} / \mathrm{Q}$ effects seem to organize the abundance variations at the onsets of some SEP events, e.g., Tylka et al. [74] Recently Ng et al. [75] developed a model of particle escape from the Alfvèn wave turbulence that energetic protons create at such a shock. Also, Zank et al. [76] have begun to model the rigidity-dependent acceleration and escape processes in a 3-dimensional model of a traveling shock. Models such as these are providing valuable insight into the SEP accelerator and may develop into more powerful tools for describing the eventto-event variations recently seen, e.g., Boberg and Tylka [77].

In addition to transport effects, the seed population available for acceleration at the $\mathrm{CME} /$ shock front includes suprathermal particles from various sources such as the solar wind, previous CME events, and ${ }^{3} \mathrm{He}$-rich impulsive flares, e.g., Mason et al. [78]. The actual mixture of suprathermal particles available for injection at the shock is unknown for any given event, but the abundance of ${ }^{3} \mathrm{He}$ may serve as a tracer for the flare contribution. The changing magnetic connection to a propagating shock implies that the observer samples the acceleration within a wide range of heliolongitudes. This time-dependent connection combines with a nonuniform mixture of source populations to create a complex picture of the source population for the SEPs.

Nevertheless, previous studies have gone ahead and averaged SEP abundances in samples of many $(>10)$ events $[79,80,81,43]$. In this sense, the SEPs might be a measure of the time-averaged and longitude-averaged abundance of the interplanetary suprathermal particles, part of which includes the fast and slow coronal flows that are sampled directly and remotely via spectroscopic methods. The SEP event-to-event variations then arise from the transport and seed population effects discussed above. Reames [43] lists such an average of 50 events and suggests that the residual Q/A effects have been averaged out to "at least an accuracy of about 10\%". Measurements of the SEP abundances will continue in the current solar maximum with more sensitive instruments on platforms such as Wind and ACE. As the new mea- 


\section{SOHO/CELIAS/MTOF}

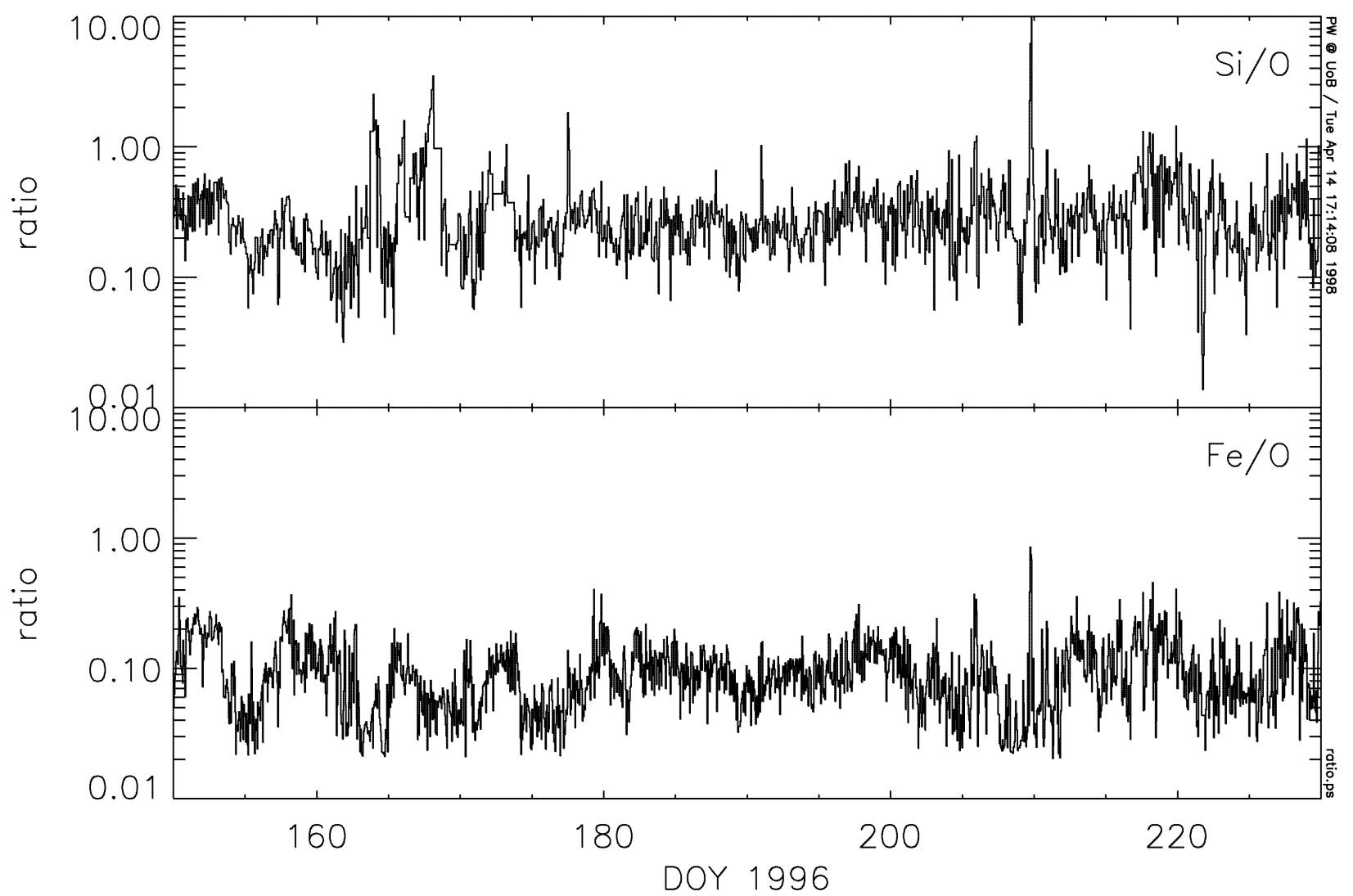

FIGURE 1. Abundance ratios of silicon and iron to oxygen. Thirty minute running averages of 5 minute data from CELIAS/MTOF aboard SOHO.

surements accumulate, we expect that new SEP abundance compilations along the lines of Reames [43] will address the issue of event-averages over a broader energy range and with high sensitivity.

Another broad class of SEP events have characteristics that suggest an origin not in interplanetary space but at a flare site. We list several of these observables here: enrichments of ${ }^{3} \mathrm{He}$ by a factor of 10-1000 compared to the solar wind; enrichments of $\mathrm{Ne}-\mathrm{Si}$ and $\mathrm{Fe}$ by factors of 3-5 and 10, respectively; association with streaming 10-100 keV electrons; scatter-free propagation; high ionization states appropriate to a 5-10 MK source. An observer at 1 AU sees the SEPs from these impulsive solar flares arrive with a velocity dispersion when there is a good magnetic connection between $1 \mathrm{AU}$ and an active region at western solar longitudes, e.g., [82, 83]. Different wave-particle resonance modes are thought to cause the large abundance enhancements of the ${ }^{3} \mathrm{He}$ and heavy ions. New measurements at 1 AU, e. g. Ho et al. [84] are revisiting the possible link between the energetic electrons and the ${ }^{3} \mathrm{He}$ enrichments.
Current instrumentation can readily resolve individual particle injections of ${ }^{3} \mathrm{He}$-rich events at $1 \mathrm{AU}$ [85], but it is difficult to tie the in-situ particles to observations of the same flare via photons. The X-ray events are often low intensity and more numerous, so correlations are difficult. The best hope for correlated optical and particle measurements lies in the broad line emissions from SEPs that precipitate into the solar atmosphere during a solar flare. Such correlations may become possible with current particle instrumentation and X-ray imaging spectroscopy from the HESSI mission [86].

\section{CONNECTION BETWEEN CORONAL AND SOLAR WIND STRUCTURES}

The general connection between fast solar wind and coronal holes is well established. Comparison of elemental abundances in streamer legs with slow solar wind abundances seems to show that the slow wind does arise from the edges (not the cores) of streamers. 
There remains a troubling question as to what exactly the streamer legs are. Observationally, they are high density, low outflow speed structures very similar to the streamer cores except in abundance relative to hydrogen. While their appearance suggests that they correspond to the open field lines closest to the closed field structures of the streamer cores, this has not been verified by sufficiently detailed field models.

The next challenge is to identify specific features in the solar wind with their sources at the Sun. Traditionally, the solar wind has been traced back to the Sun by means of simple ballistic outflow models. More recently, global magnetic field models and MHD outflow solutions have been applied. Ko et al. [87] present a promising attempt to relate solar wind properties measured with ACE/SWICS to structures observed by UVCS at several heights based on a 3-D MHD code. The energetic particles measured within magnetic clouds originate in impulsive flares (i.e., ${ }^{3} \mathrm{He}$-rich and $\mathrm{Fe} / \mathrm{O} \sim 1$ ). If cases can be found where a shock forms in the corona and rapidly weakens, it may eventually be possible to infer the region (e.g., legs of coronal streamers, etc.) where they were injected.

An exciting prospect is the measurement of energetic particles within Magnetic Clouds. Energetic particles inside MCs that are not associated with IP shocks (or with very weak ones), should show composition rather the same as that in the solar corona. Thus they would indicate the region (legs of coronal streamers, etc.) from which they were injected inside the CME. Moreover, their ${ }^{3} \mathrm{He}$ and $\mathrm{Fe}$ abundances could provide additional information about the problematic flare-CME connection.

\section{REFERENCES}

1. Kohl, J.L. et al. 1997, Sol. Phys. 175, 613

2. Ofman, L. 2000, submitted to ApJ

3. Sheeley, N.R. Jr., 1996, ApJ, 469, 423.

4. Del Zanna, G., Bromage, B.J.I., and Mason, H.E., 2001, this issue.

5. Gabriel, A.H. 1971, Sol. Phys., 21, 392

6. Schrijver, C.J., and McMullin, R.A. 2000, ApJ, 531, 1121

7. Wood, K., and Raymond, J. 2000, ApJ, 540, 563

8. Mason, H.E., 1992, ESA SP-348, 297

9. Phillips, K. J. H., 1997, Adv. Space Res. 20, 79.

10. Young, P.R., Landi, E., Thomas, R.J., 1998, A\&A 329, 291

11. Eissner, W., Jones, M., Nussbaumer, H., 1974,

Comp.Phys.Comm., 8, 270

12. Hibbert, A., 1975, Comp.Phys.Comm., 9, 141

13. Eissner, W., Seaton, M.J., 1972, J.Phys.B, 5, 2187

14. Burgess, A., Sheorey, V.B., 1974, J.Phys.B, 7, 2403

15. Burke, P.G., Hibbert, A., Robb, W.D., 1971, J.Phys.B, 4, 153

16. Dere, K.P., Landi, E., Mason, H.E., Monsignori Fossi, B.C., Young, P.R., 1997, A\&ASS, 125, 149
17. Smith, R.K., Brickhouse, N.S., Lieddahl, D.A., and Raymond, J.C. 2001, R, ApJL, in press

18. Dere, K.P., Landi, E., Young, P.R., and Del Zanna, G., 2001, ApJSS, 134, 331.

19. Binello, A.M., Landi, E., Mason, H.E., Storey, P.J., Brosius, J.W., 2001, A\&A, 370, 1071

20. Storey, P.J., Mason, H.E., Young, P.R., 2000, A\&AS, 141, 285

21. Storey, P.J. and Zeippen, C.J., 2001, MNRAS, 324, L7

22. Smith, B.W., Mann, J.B., Cowan, R.D., and Raymond, J.C. 1985 , ApJ, 298, 898

23. Thomas, R.J., Neupert, W.M., 1994, ApJSS, 91, 461

24. Brickhouse, N.S., Raymond, J.C., and Smith, B.W. 1995, ApJS, 97, 551

25. Landi, E., 1998, PhD Thesis - University of Florence

26. Del Zanna, G., 1999, PhD Thesis - University of Central Lancashire.

27. Landi, E., Feldman, U., 2001, ApJ, in preparation

28. Young,P.R. and Mason H.E., 1998, SSRv. 85, 315.

29. Wilhelm, K., et al., 1995, Solar Phys. 162, 189

30. Akmal, A. Raymond, J.C., Vourlidas, A., Thompson, B., Ciaravella, A., Ko, Y.-K., Uzzo, M., and Wu, R. 2001, ApJ, 553,922

31. Mazzotta, P., Mazzitelli G., Colafrancesco S., Vittorio N., 1998, A\&AS 133, 403

32. Masai, K., 1997, A\&A, 324, 410

33. Phillips, K.J.H., Feldman, U., 1997, ApJ, 477, 502

34. Arnaud, M., Rothenflug, R., 1985, A\&AS 60, 425

35. Allen, R., Landi, E., Landini, M., Bromage, G.E., 2000, A\&A, 358, 332

36. Shull, J.M., Van Steenberg, M., 1982, ApJS 48, 95 and ApJS 49, 351

37. Arnaud, M., Raymond, J.C., 1992, ApJ 398, 394

38. Gianetti, D., Landi, E., Landini, M., 2000, A\&A, 360, 1148

39. Gloeckler, G., Geiss, J., Balsiger, H., Bedini, P., Cain, J.C., Fisher, J., Fisk, L.A., Galvin, A.B., Gliem, F., and Hamilton, D.C., 1992 A \& A Suppl., 92, 267

40. Hovestadt, D., et al. 1995, Solar Phys., 162, 441

41. Gloeckler, G., Bedini, P., Bochsler, P., Fisk, L.A., Geiss,

J., Ipavich, F.M., Cani, J., Fischer, J., Kallenbach, R., Miller,

J., Tums, O., and Winner, R. 1998, SSRv, 86, 495

42. Wimmer-Schweingruber, R. 1994, PhD Thesis, University of Bern

43. Reames, D.V. 1999, SSRv, 90, 413

44. Stone, E.C., et al., 1998, SSRv, 86, 1

45. Grevesse, N., and Sauval, A.J. 1998, SSRv, 85, 161

46. Fludra, A., and Schmelz, J.T. 1999, A\& A 348, 286

47. Del Zanna, G., and Bromage, B.J.I. 1999, SSRv, 87, 169

48. Raymond, J.C., et al. 1997, Sol. Phys., 175, 645

49. Parenti, S., Bromage, B.J.I., Poletto, G., Suess, S., Raymond,J.C., Noci, G., and Bromage, G.E. 2001, this volume

50. Zangrilli, L., Poletto, G., and Biesecker, D. 2000, this volume

51. Marrocchi, D., Antonucci, E., and Giordano, S. 2001, Annales Geophysicae, 19, 135

52. Teriaca, L., Poletto, G., Falchi, A., and Doyle, J.G. 2001, this volume

53. Antonucci, E., Giordano, S., and Marocchi, D. 2001, this volume

54. Laming, J.M., and Feldman, U. 2001, this volume

55. Doschek, G.A., Laming, J.M., Fledman, U., Wilhelm, K., 
Lemaire, P., Schüle, U., and Hassler, D.M. 1998, ApJ, 504, 573

56. Feldman, U., Schüle, U., Widing, K.G., and Laming, J.M. 1998, ApJ, 505, 999

57. Young, P.R., Klimchuk, J.A., and Mason, H.E. 1999, A\& A, 350,286

58. Warren, H.P. 1999, Sol. Phys., 190, 363

59. Laming, J.M., Feldman, U., Drake, J.J., and Lemaire, P. 1999, ApJ, 518, 926

60. Feldman, U., Doschek, G.A., Schüle, U., and Wilhelm, K. 1999, ApJ, 518, 500

61. Parenti, S., Bromage, B.J.I., Poletto, G., Noci, G., Raymond, J.C., and Bromage, G.E. 2000, A\& A, 363, 800

62. Rank, G., Czaykowska, A., Bagalá, L.G., and Haerendel, G. 1999, ESA-SP-448, p. 349

63. Dwivedi, B.H., Curdt, W., and Wilhelm, K. 1999, ApJ, 517,516

64. Ko, Y.-K., Raymond, J.C., Li, J., Ciaravella, A., Michels, J., Fineschi, S., and Wu, R. 2001, in preparation

65. Ciaravella, A., et al. 1997, ApJL, 491, 59

66. Spicer, D. Feldman, U., Widing, K.G., and Rilee, M. 1998, ApJ, 494, 450

67. Feldman, W.C., Asbridge, J.R., Bame, S.J., and Gosling, J.T. 1978, JGR, 83, 2177

68. Geiss, J., Gloeckler, G., von Steiger, R., Balsiger, H., Fisk, L.A., Galvin, A.B., Ipavich, F.M., Livi, S., McKenzie, J.F., Ogilvie, K.W., and Wilken, B. 1995, Science, 268, 1033

69. Neugebauer, M., 1981, in Fundamentals of Cosmic Physics, Gorden and Breach Science Publishers, Inc., 1981, 7, 131-199.

70. Wurz, P., Aellig, M.R., Bochsler, P., Hefti, S., Ipavich, F.M., Galvin, A.B., Gruenwaldt, H., Hilchenbach, M., Gliem, F., Hovestadt, D. 1999, Phys. Chem. Earth (C), 24(4), 421

71. Geiss, J., Gloeckler, G., and von Steiger, R. 1995, SSRv, 72,49

72. Aellig, M.R., Hefti, S., Grünwaldt, H., Bochsler, P., Wurz, P., Ipavich, F.M., Hovestadt,D. 1999, JGR, 104, 24769

73. Hefti, S., Grünwaldt, H., Ipavich, F.M., Bochsler, P., Hovestadt, D., Aellig, M.R., Hilchenbach, M., Kallenbach, R., Galvin, A.B., Geiss, J., Gliem, F., Gloeckler, G., Klecker, B., Marsch, E., Moebius, E., Neugebauer, M., Wurz,P. 1998, JGR, 103, 29697

74. Tylka, A. J., Boberg, P.R., McGuire, R.E., Ng, C.K., and Reames, D.V. 2000, Acceleration and Transport of Energetic Particles Observed in the Heliosphere, ed. R. A. Mewaldt et al., AIP Conf. Proc., 528, 147

75. Ng, C. K. et al. 1999, Geophys. Res. Lett. 26, 2145

76. Zank, G. P., Rice, W.K.M., and Wu, C.C. 2000 , J. Geophys. Res. 105, 25079

77. Boberg, P. R., and Tylka, A.J. 2000, Acceleration and Transport of Energetic Particles Observed in the Heliosphere, ed. R. A. Mewaldt et al., AIP Conf. Proc., 528, 115

78. Mason, G. M., Mazur, J.E., and Dwyer, J.R. 1999, ApJL, 525, L133

79. Cook, W. R., Stone, E.C., and Vogt, R.E. 1984, ApJ, 279, 827

80. Breneman, H. H. and E. C. Stone 1985, ApJL, 299, L57

81. Mazur, J. E. et al. 1993, ApJ, 404, 810

82. Kahler, S. W. et al. 1987, Sol. Phys., 107, 385

83. Mazur, J. E. et al. 2000, ApJL, 532, L79

84. Ho, G.C., Roelof, E.C., Hawkins, S.E. III, Gold, R.E., Mason, G.M., Dwyer, J.R., and Mazur, J.E. 2001, Astrophys.
J.,552, 863

85. Mason, G. M., Dwyer, J.R., and Mazur, J.E. 2000, ApJL, $545, \mathrm{~L} 157$

86. Lin, R. P. 2000, in High Energy Solar Physics: Anticipating HESSI, ASP Conf. Proc. 206, 1

87. Ko, Y.-K., Zurbuchen, T., Strachan, L., Riley, P., and Raymond, J.C., this volume 